\title{
EFECTOS DE LA COMBUSTIÓN A LEÑA EN LA CALIDAD DEL AIRE INTRADOMICILIARIO. LA CIUDAD DE TEMUCO COMO CASO DE ESTUDIO
}

Alejandra Cortés ${ }^{1}$ e Ian Ridley²

\section{Introducción}

La Organización Mundial de la Salud ${ }^{3}$ ha declarado en sus estudios que la mitad de la población mundial depende de combustibles sólidos, como la madera, el carbón, residuos agrícolas o estiércol, para satisfacer sus necesidades

1 Chile. Arquitecta, Universidad de Chile. MSc Environmental Design and Engineering, University College London, Reino Unido. MA Arquitectura y Medioambiente, Universidad Politécnica de Cataluña, España. Profesora Asistente, Facultad de Arquitectura y Urbanismo, Universidad de Chile. Correo electrónico: acortesfuentes@uchilefau.cl.

2 Australia. PhD University of Westminster. Dr. at School of Property Construction and Project Management, RMIT University, Melbourne, Australia. Senior Lecturer in Environmental Design and Engineering in the Bartlett School of Graduate Studies, University College London. Correo electrónico: i.ridley@ucl.ac.uk. energéticas básicas. La energía de biomasa, que incluye la leña, residuos de cultivos y desechos de animales, provee en promedio cerca del 30\% de la energía primaria en los países en desarro$1 \mathrm{l}^{4}$. Más de dos mil millones de personas dependen directamente de la biomasa como principal o única fuente de energía.

4 Matthews et al, 2000. 
Cocinar y calefaccionar con combustibles sólidos en fuegos abiertos o cocinas tradicionales genera altos niveles de contaminación del aire dentro de los hogares. A nivel global, la contaminación intradomiciliaria produce alrededor de 1,5 millones de muertes al año ${ }^{5}$, afecta a casi la mitad de la población mundial y es responsable de aproximadamente el $4 \%$ de la carga de enfermedades expresadas en AVAD $^{6}$-años de vida ajustados por discapacidad-. La población afectada es predominantemente rural y de bajos ingresos, y los más vulnerables son las mujeres, niños y ancianos.

De acuerdo a la evidencia epidemiológica, en 2005 la Organización Mundial de la Salud (OMS) publicó las Directrices de Calidad del Aire (Air Quality Guidelines) con un resumen de la evaluación del riesgo asociado a la concentración de contaminantes, que también puede ser aplicado a la contaminación de ambientes interiores $^{7}$. El documento presenta una orientación numérica y metas intermedias de reducción a la exposición a largo y corto plazo de concentración de diferentes contaminantes, entre ellos el material particulado ${ }^{8}$ MP10 y MP2.5 (ver Tabla 1, con algunas de la metas de la OMS).

5 World Health Organization, 2006.

6 World Health Organization, 2000.

7 World Health Organization, 2005.

8 MP significa Material Particulado; 10 y 2.5 es el tamaño de la partícula que es expresada en términos de su tamaño aerodinámico; su unidad de medida es $\mu \mathrm{g} / \mathrm{m}^{3}$ : microgramo por metro cúbico de aire, y representa la concentración de los contaminantes.
Las directrices se basan en las extensas pruebas científicas relativas a la contaminación del aire y sus consecuencias para la salud.

El único combustible que se considera como emisor de material particulado por concepto de calefacción es la leña. Otros combustibles derivados del petróleo, tales como el gas licuado, el petróleo para calefacción, el kerosene doméstico y el gas natural, se consideran con cero emisiones de material particulado?.

Para clasificar el material particulado existen dos métricas comúnmente utilizadas: partículas menores a 10 micrones (MP10) y partículas menores a 2,5 micrones (MP2.5). En el MP10 se pueden distinguir dos fracciones: la gruesa, es decir, entre 2,5 y 10 micrones y la fina, menor a 2,5 micrones $^{10}$. Mientras más pequeño es el tamaño de la partícula, más profundamente puede entrar en los pulmones y sistema respiratorio, acarreando toxinas por el cuerpo a través de la sangre.

La OMS ${ }^{11}$ reconoce que las mediciones in situ de contaminantes intradomiciliarios son técnicamente difíciles, ya que involucran equipos relativamente costosos, cuidadosos procedimientos y controles de calidad. La medición de la exposi-

9 Ambiente Consultores, 2007a.

10 Ministerio del Medio Ambiente, 2012a.

11 World Health Organization, 2005. 
TABLA 1: DIRECTRICES DE CALIDAD DEL AIRE PARA MATERIAL PARTICULADO MP10 Y MP2.5.

\begin{tabular}{|c|c|c|c|}
\hline \multicolumn{4}{|c|}{ Concentración Media Anual ( $\mu \mathrm{g} / \mathrm{m} 3)$} \\
\hline & MP10 & MP2.5 & Fundamentos \\
\hline $\begin{array}{l}\text { Meta In- } \\
\text { termedia } 1\end{array}$ & 70 & 35 & $\begin{array}{l}\text { Estos niveles de concentración de material particulado están asociados con un } \\
15 \% \text { de mayor riesgo de mortalidad a largo plazo, comparado al nivel de la Directriz } \\
\text { de Calidad del Aire. }\end{array}$ \\
\hline $\begin{array}{l}\text { Directriz } \\
\text { de Calidad } \\
\text { del Aire }\end{array}$ & 20 & 10 & $\begin{array}{l}\text { Este es el nivel más bajo de concentración de material particulado. Se ha demos- } \\
\text { trado que la mortalidad por cáncer aumenta, con una confiabilidad del } 95 \% \text { en rela- } \\
\text { ción a la exposición de MP2.5 a largo plazo. }\end{array}$ \\
\hline \multicolumn{4}{|c|}{ Concentración en 24 horas $\left(\mu \mathrm{g} / \mathrm{m}^{3}\right)$} \\
\hline & MP10 & MP2.5 & Fundamentos \\
\hline $\begin{array}{l}\text { Meta In- } \\
\text { termedia } 1\end{array}$ & 150 & 75 & $\begin{array}{l}\text { Alrededor de un } 5 \% \text { de aumento en mortalidad a corto plazo, comparado con la } \\
\text { Directriz de Calidad del Aire. Información basada en los coeficientes de riesgo publi- } \\
\text { cados por estudios de multi-centros y meta-análisis. }\end{array}$ \\
\hline $\begin{array}{l}\text { Directriz } \\
\text { de Calidad } \\
\text { del Aire }\end{array}$ & 50 & 25 & $\begin{array}{l}\text { Información basada en la relación de los niveles de material particulado de } 24 \text { horas } \\
\text { y la media anual. }\end{array}$ \\
\hline
\end{tabular}

Fuente: Organización Mundial de la Salud (WHO 2005)

ción de las personas agrega aún más dificultades ${ }^{12}$. Estas complejas metodologías constituyen una de las causas por las cuales las mediciones directas de la exposición de las personas a contaminantes dentro de los hogares han sido menos realizadas y estudiadas que las mediciones de contaminantes atmosféricos al aire libre.

Dadas las dificultades de las mediciones in situ, el objetivo de esta investigación es predecir posibles

12 World Health Organization, 2000. escenarios de exposición de ocupantes a material particulado dentro de los hogares y su potencial riesgo a la salud. Para ello se usan software de simulación como herramienta metodológica de investigación. El caso de estudio es la ciudad de Temuco, ya que fue declarada zona saturada de MP10 en 2005 y hasta entonces no existían precedentes en el país en el cual la principal fuente de emisiones fuera la combustión residencial de leña para cocinar y calefaccionar los hogares. 


\section{Contexto nacional}

En Chile, el 20\% de la energía primaria consumida proviene de la leña, que es la segunda fuente de energía más importante después del petróleo ${ }^{13}$. De esto, un 59\% corresponde a consumo residencial de leña ${ }^{14}$. Del total del consumo energético en el país, el residencial es responsable del 20\%, y por uso final éste se puede subdividir en $15 \%$ para agua sanitaria, $37 \%$ para electricidad y $52 \%$ para calefacción ${ }^{15}$.

La crisis energética mundial, como resultado del incremento del precio del petróleo y los cortes de suministro de gas importado desde Argentina, que comenzaron en 2003, ha causado el aumento del consumo de leña en la zona sur del país, especialmente en la población de menores recursos, causando a su vez el aumento de la contaminación ambiental urbana ${ }^{16}$. El mercado de la leña genera 200 millones de dólares al año, de los cuales el $35 \%$ se puede atribuir a productores rurales.

Al sur de la latitud $38^{\circ}$, más del $80 \%$ de los hogares urbanos y casi el 100\% de los hogares rurales consumen leña, probablemente porque es entre 4 y 7 veces más barata que otras fuentes de energía. Su principal uso es para cocinar y calefaccionar los

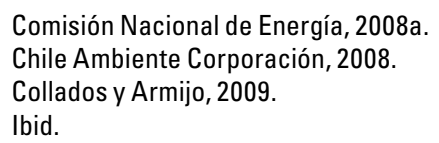

hogares ${ }^{17}$. El consumo de leña urbano del sector residencial puede alcanzar, en promedio, más de 500 mil metros cúbicos al año.

Hoy en día, el uso de biomasa y leña permite la diversificación de los recursos energéticos y reduce la dependencia de las importaciones de combustibles, que tienen precios volátiles e inciertos. Sin embargo, también la combustión de leña húmeda en aparatos ineficientes es responsable de la polución atmosférica en muchas ciudades del sur del país, generando serios problemas en la salud de la población ${ }^{18}$. A pesar de los bien conocidos impactos ambientales de la combustión a leña, su bajo costo en comparación con otros combustibles, así como la tradición cultural de su uso en las ciudades del sur del país, dificultan su sustitución.

Al respecto, en julio de 2012 entró en vigencia la norma ${ }^{19}$ que establece los máximos permitidos de emisión de material particulado para los artefactos que combustionen o puedan combustionar leña y derivados de la madera. La norma sobre calefactores, que entra en vigencia el $1^{\circ}$ de octubre de 2013 , apunta principalmente a una mejora tecnológica de los equipos que se comercializan en el país, con factores de menor emisión y mayor eficiencia.

17 Universidad de Chile, 2005.

18 Comisión Nacional de Energía, 2008b.

19 Ministerio del Medio Ambiente, 2012b. 


\section{Ciudad de Temuco como caso de estudio}

La ciudad de Temuco se ubica en el $38^{\circ} 46^{\prime}$ latitud sur. Está clasificada como zona 5 en la reglamentación térmica chilena ${ }^{20}$, lo que significa que los grados días anuales para calefacción ${ }^{21}$ oscilan entre 1250-1500. La ciudad presenta temperaturas promedio cercanas a los $12{ }^{\circ} \mathrm{C}$ y precipitaciones sobre $1.000 \mathrm{~mm}$ al año. Las temperaturas extremas absolutas pueden variar entre los $-5^{\circ} \mathrm{C}$ (junio) y $37{ }^{\circ} \mathrm{C}$ (febrero). Estas temperaturas extremas en los meses invernales son las que hacen particularmente intensivo el uso de calefacción residencial en Temuco ${ }^{22}$.

La ciudad fue declarada zona saturada de material particulado MP10 en 2005. En Chile, la norma primaria de calidad del aire para MP10 y MP2.5 establece concentraciones máximas permitidas en $24 \mathrm{hrs}$ y anualmente. Se entiende sobrepasada la norma diaria de MP10 cuando el percentil 98 es mayor o igual que $150 \mu \mathrm{g} / \mathrm{m}^{3}$, o cuando en el periodo de un año hay más de 7 días con concentraciones superiores a ese valor.

20 Ministerio de Vivienda y Urbanismo, 2006.

21 Los grados/día de calefacción están directamente relacionados con las demandas de energía que la vivienda requiere en el período considerado para lograr la temperatura interior base $\left(15^{\circ} \mathrm{C}\right)$.

22 Ministerio Secretaría General de la Presidencia, 2010.
En la ciudad de Temuco, el 75\% de la leña es usada en invierno; el 35\% de los hogares tienen una cocina a leña, y el 30\% una estufa de combustión lenta. En promedio, el consumo de leña en la ciudad es de 5,8 metros cúbicos anuales y el $48 \%$ del consumo se concentra en la población con menores ingresos $^{23}$. De acuerdo al Censo ${ }^{24} 2002$, el 16\% de los hogares usa leña para cocinar y calefaccionar. Del total de las emisiones de MP10, el $87 \%$ es atribuido al consumo residencial de leña.

En mayo de 2010 fue aprobado legalmente el Plan de Descontaminación Atmosférica (PDA), que permite poner en práctica acciones para la reducción de las emisiones contaminantes. El objetivo del plan es reducir en un plazo de 10 años el 31\% de las emisiones y así dar cumplimiento a la norma primaria de calidad ambiental para material particulado respirable MP10, sacando a la ciudad de su condición de zona saturada ${ }^{25}$. El PDA reconoce que hay cuatro factores que han contribuido a que la principal fuente de emisión de material particulado sea la combustión de leña residencial:

1. Comercialización y uso de leña que no cumple con los estándares mínimos de calidad.

\section{Universidad de Chile, 2005}

Instituto Nacional de Estadísticas, 2012.

Ministerio Secretaría General de la Presidencia, 2010. 
2. Uso de equipos ineficiente de combustión a leña.

3. Alta demanda de leña para calefacción debido a la pobre aislación térmica de las viviendas.

4. Comportamiento de los consumidores de leña: no adquieren leña seca y no operan los artefactos de manera adecuada y eficiente.

En relación a la combustión residencial, las principales acciones del PDA son:

1. Regulación referida al uso y mejoramiento de la calidad de la leña: la leña deberá cumplir con los estándares establecidos por el Sistema Nacional de Certificación de Leña ${ }^{26}$. Esta iniciativa tiene por objetivo reducir la deforestación y la polución ambiental y controlar el mercado informal de la leña.

2. Regulación referida al mejoramiento de la eficiencia térmica de la vivienda: a través del Ministerio de Vivienda y Urbanismo, con el Subsidio para Acondicionamiento Térmico de la Vivienda, que permite mejorar la aislación térmica de viviendas sociales.

3. Regulación referida al uso y mejoramiento de la calidad de los artefactos residenciales que usan a leña: se realiza mediante la certificación

26 Sistema Nacional de Certificación de Leña, 2012. de los artefactos comercializados en la ciudad y la promoción del recambio voluntario, que compromete subsidios del gobierno.

Algunos estudios anticipan que el recambio de la cocina a leña en los hogares de menores recursos puede presentar mayores problemas, esencialmente porque es usada tanto para cocinar como para calefaccionar es decir, su remplazo implicaría la adquisición de dos nuevos artefactos, aumentando el gasto familiar en combustible. De acuerdo con las proyecciones económicas, los resultados indican que a pesar del subsidio, los ahorros en costos de operación son bajos e incluso negativos para los grupos de menores ingresos ${ }^{27}$. Además, la cocina a leña presenta algunas ventajas que son más difíciles de cuantificar, como su uso para contar con agua caliente y para secar ropa. Tiene fuertes raíces culturales, pues se estima que el uso de la cocina a leña no corresponde a los típicos factores económicos de mercado - precio, costo, competitividad-sino para mantener hábitos tradicionales ${ }^{28}$.

La hipótesis de este estudio se basa en que si se aplica el subsidio de acondicionamiento térmico, las viviendas pueden quedar más herméticas y menos permeables en relación a su nivel de

27 Universidad de la Frontera, 2009

28 Ambiente Consultores, 2007b. 
infiltraciones de aire ${ }^{29}$. Si esto sucede y la cocina a leña no es remplazada -como se puede predecir para grupos más vulnerables-, entonces es posible que la concentración de contaminantes dentro de los hogares aumente, empeorando la calidad del aire intradomiciliario y afectando la salud de las personas. Por otra parte, aunque aumenten las temperaturas interiores por la eficiencia térmica de la envolvente de la vivienda, los hábitos de quema de leña probablemente no cambiarán, porque la cocina requiere cierta cantidad de producción de calor para hervir agua o cocinar, como también para otros propósitos.

\section{Metodología}

Esta investigación analiza, a través de simulaciones computacionales, la calidad del aire intradomiciliario en viviendas de Temuco, causada por las emisiones de material particulado de una cocina a leña. Ello se realiza considerando diferentes escenarios de permeabilidad y tasas de renovación de aire en un caso específico, de modo de predecir la exposición de los ocupantes y sus riesgos de salud.

29 Las infiltraciones de aire es el paso del aire no controlado a través de ranuras de ventanas, puertas, agujeros en las paredes, techos, etc. Éstas son más notorias en las fachadas expuestas al viento, debido a la presión que ejerce éste sobre las aberturas. El rendimiento energético de la vivienda se ve afectado en forma importante por las infiltraciones. Una vivienda que no esté sellada puede necesitar hasta un $30 \%$ más de energía que una vivienda relativamente hermética.
El objetivo principal de este estudio es evaluar los cambios de concentración de contaminantes y su distribución por recinto, cuando la permeabilidad de la vivienda se modifica, pero las tasas de emisión de material particulado de la cocina a leña se mantienen iguales. Para ello se usaron dos modelos: TAS, un software de simulación dinámica de comportamiento térmico de edificios, para predecir las temperaturas interiores de la vivienda; y CONTAM, un software de ventilación y de modelamiento de calidad del aire, para predecir la concentración de MP10 y MP2.5 en cada habitación.

Los escenarios de permeabilidad de las viviendas se estimaron a partir de una investigación realizada por la Universidad de la Frontera ${ }^{30}$, en la cual se efectuaron test de infiltraciones ${ }^{31}$ a varias viviendas en la ciudad de Temuco. Una muestra de 24 viviendas se toma como base para realizar los modelos de esta investigación y se definen así 3 escenarios de permeabilidad:

1. Permeabilidad $30 \mathrm{~m}^{3} / \mathrm{m}^{2} / \mathrm{h} @ 50 \mathrm{~Pa}$ : promedio de las 8 casas con mayor permeabilidad, que representa el 33\% de la muestra testeada.

\section{Cárdenas, 2010.}

31 La unidad usada en este test son metros cúbicos de aire, que pasan por metro cuadrado de envolvente, en una hora a una diferencia de 50 pascales: $\mathrm{m}^{3} / \mathrm{m}^{2} / \mathrm{h} @ 50 \mathrm{~Pa}$. 


\section{FIGURA 1: VIVIENDA A MODELAR}

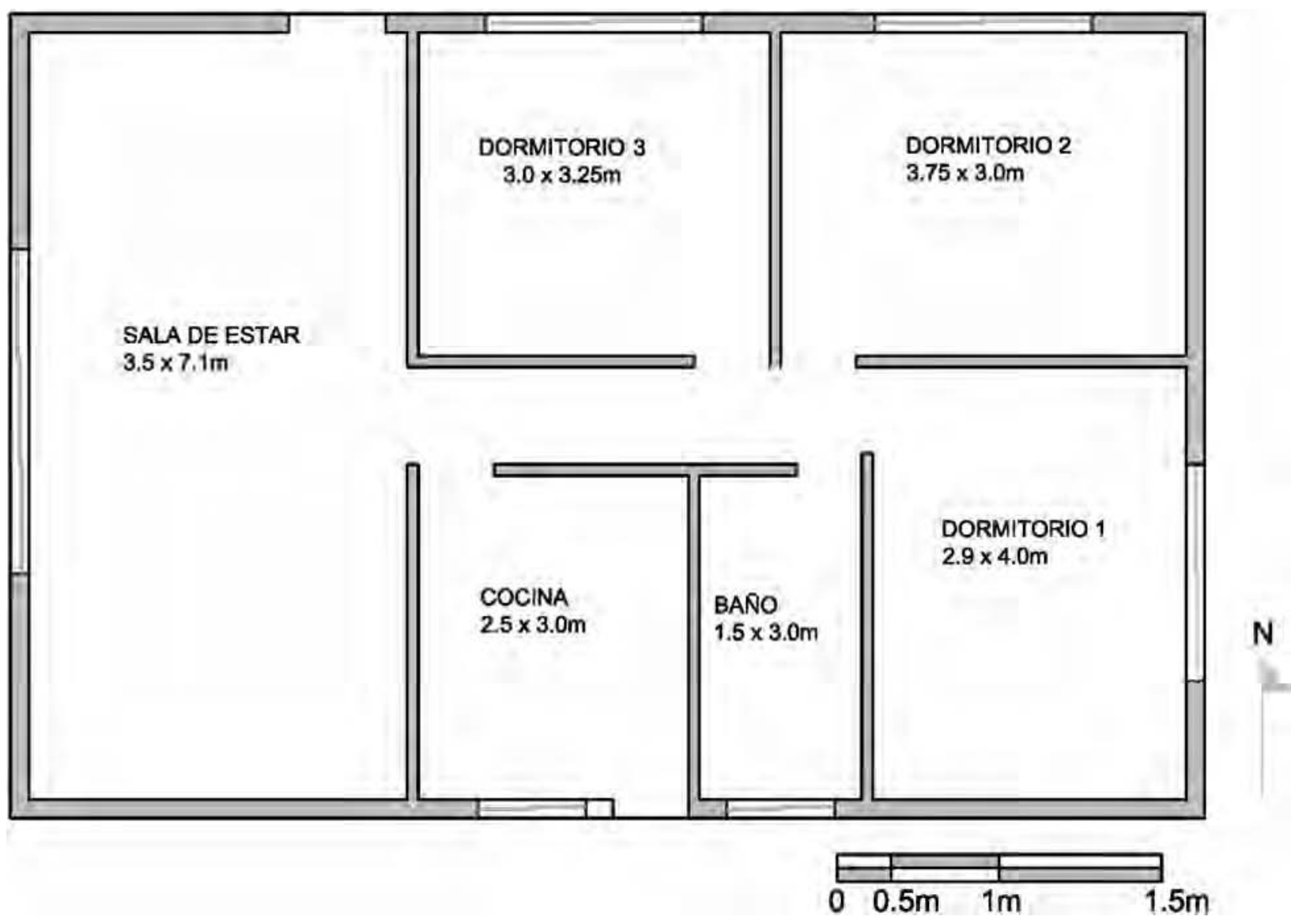

Fuente: Elaboración propia. 
FIGURA 2. PROMEDIO DE HORARIO EN INVIERNO DE CONCENTRACIÓN DE MP ${ }_{10}$ EN SALA DE ESTAR, BAJO DIFERENTES ESCENARIOS DE PERMEABILIDAD.

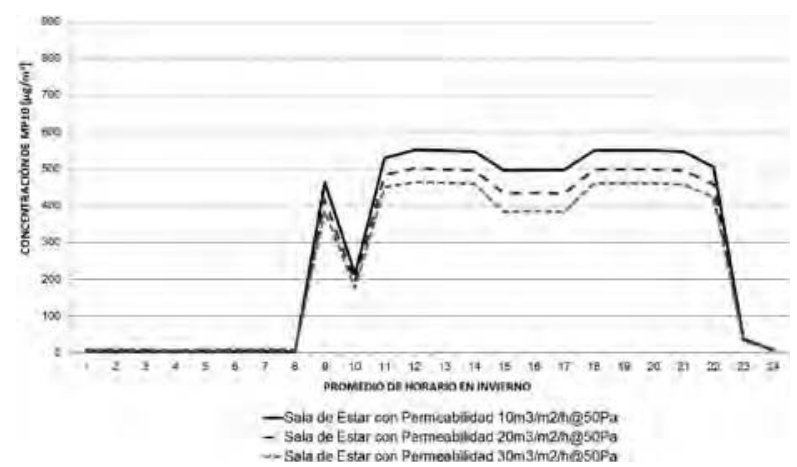

Fuente: Elaboración propia

FIGURA 4. PROMEDIO DE HORARIO EN INVIERNO DE CONCENTRACIÓN DE MP ${ }_{2.5}$ EN SALA DE ESTAR, BAJO DIFERENTES ESCENARIOS DE PERMEABILIDAD.

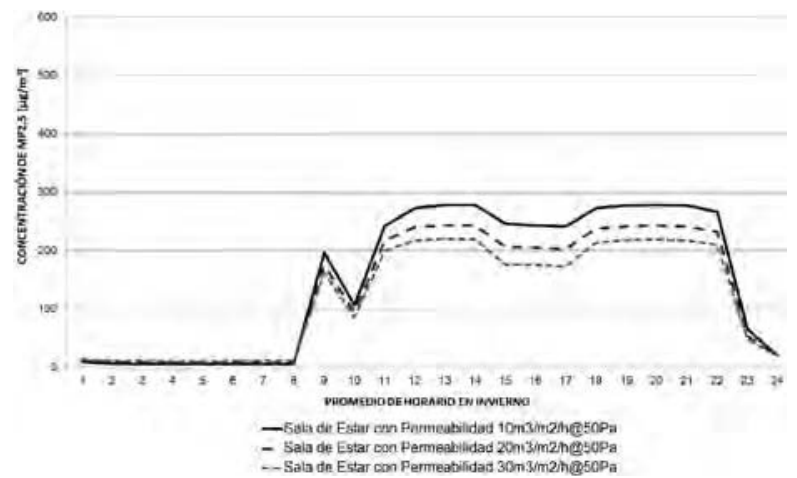

Fuente: Elaboración propia

OPINIÓN: Efectos de la combustión a leña en la calidad del aire intradomiciliario. La ciudad de Temuco como caso de estudio / Alejandra Cortés e lan Ridley
FIGURA 3. PROMEDIO DE HORARIO EN INVIERNO DE CONCENTRACIÓN DE MP ${ }_{10}$ EN COCINA, BAJO DIFERENTES ESCENARIOS DE PERMEABILIDAD

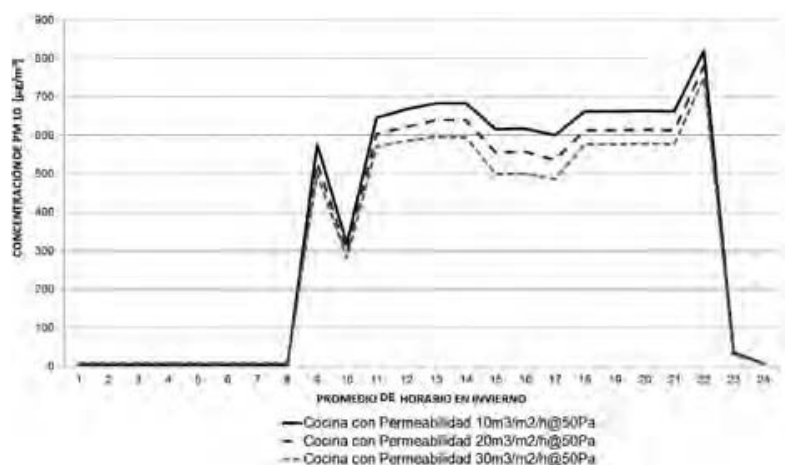

Fuente: Elaboración propia.

FIGURA 5. PROMEDIO DE HORARIO EN INVIERNO DE CONCENTRACIÓN DE MP ${ }_{2.5}$ EN COCINA, BAJO DIFERENTES ESCENARIOS DE PERMEABILIDAD.

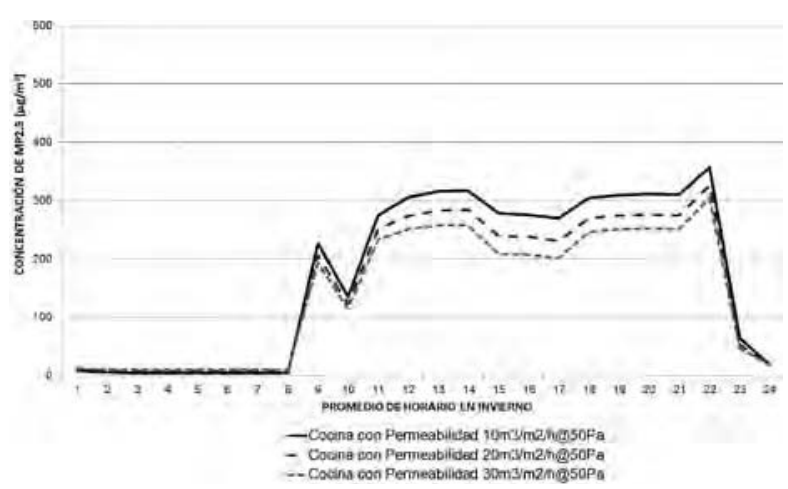

Fuente: Elaboración propia.

revista invi ํㅜ 78 / Agosto 2013 / Volumen № 28: 257-271 265 
2. Permeabilidad de $20 \mathrm{~m}^{3} / \mathrm{m}^{2} / \mathrm{h} @ 50 \mathrm{~Pa}$ : promedio de las 8 casas que están en el centro de la distribución estadística y que representan el promedio de la muestra testeada.

3. Permeabilidad de $10 \mathrm{~m}^{3} / \mathrm{m}^{2} / \mathrm{h} @ 50 P a$ : se asume como el nivel alcanzado una vez que la vivienda es refaccionada con el subsidio de reacondicionamiento térmico.

Las características físicas de la vivienda a modelar (ver Figura 1) son las de una casa aislada de madera (50\% del parque construido en Temuco) y el diseño es una variable que se deja fija en las simulaciones. Se asume como una vivienda con materiales y sistemas constructivos usados antes de 2000, es decir sin aislación térmica ${ }^{32}$, ya que la mayor parte del parque de viviendas de Temuco fue construido antes de esa fecha ${ }^{33}$. Además, se asume un horario específico de ventilación que se mantiene igual para ambos software. Cabe destacar que esta investigación no intenta reproducir el comportamiento de las personas, sino comparar bajo criterios uniformes cómo distintos escenarios de permeabilidad afectan la concentración de contaminantes dentro de los hogares.

32 Año en que se implementa la primera parte de la regulación térmica de viviendas, a través de la Ordenanza General de Urbanismo y Construcción.

33 Ambiente Consultores, 2007a.

\section{Resultados}

Todas las simulaciones se realizaron entre los meses de abril y septiembre, considerados los más fríos en la ciudad y cuando las concentraciones de material particulado en la atmósfera aumentan. Los resultados arrojaron concentraciones mayores de material particulado en la cocina y sala de estar, que en el resto de la vivienda. Si analizamos estos dos espacios y las concentraciones de MP10, con los tres escenarios de permeabilidad, se puede observar (ver figuras 2 y 3) que las mayores diferencias se producen después del período de ventilación (entre 9:00 - 10:00 hrs), cuando la casa ya no está expuesta a la ventilación natural por ventanas. En la cocina, las concentraciones de MP10 están en un rango de entre 500-700 $\mathrm{\mu g} / \mathrm{m}^{3}$, mientras que en la sala de estar se sitúan entre $400-600 \mu \mathrm{g} / \mathrm{m}^{3}$.

Las concentraciones de MP2.5 (ver figuras 4 y 5) siguen el mismo patrón que las concentraciones de MP10. En la cocina varían entre $200-350 \mu \mathrm{g} / \mathrm{m}^{3}$, mientras que en la sala de estar entre $180-280 \mu \mathrm{g} / \mathrm{m}^{3}$. 
TABLA 2. PROMEDIO DE CONCENTRACIÓN DE MATERIAL PARTICULADO PARA EL PERÍODO DE INVIERNO.

\begin{tabular}{llll} 
Concentración de & \multicolumn{1}{l}{ Escenarios de Permeabilidad $\left(\mathrm{m}^{3} / \mathrm{m}^{2} / \mathrm{h} @ 50 \mathrm{~Pa}\right)$} & \\
Contaminante $\left(\mu \mathrm{g} / \mathrm{m}^{3}\right)$ & 10 & 20 & 30 \\
\hline $\mathrm{MP}_{10}$ & 123,4 & 118,1 & 113,0 \\
\hline $\mathrm{MP}_{2.5}$ & 64,2 & 60,9 & 58,0 \\
\hline
\end{tabular}

Fuente: Elaboración propia

Estos resultados muestran que los máximos de concentración de MP10, en cocina y sala de estar, son del orden de $100 \mu \mathrm{g} / \mathrm{m}^{3}$ más altos en la vivienda simulada con permeabilidad $10 \mathrm{~m}^{3} / \mathrm{m}^{2} /$ h@50Pa, que aquella con permeabilidad $30 \mathrm{~m}^{3} /$ $\mathrm{m}^{2} / \mathrm{h} @ 50 \mathrm{~Pa}$. De igual manera, el promedio diario de concentración de MP10 es $10 \mu \mathrm{g} / \mathrm{m}^{3}$ más alto en la vivienda simulada con permeabilidad $10 \mathrm{~m}^{3}$ / $\mathrm{m}^{2} / \mathrm{h} @ 50 \mathrm{~Pa}$ versus aquella con permeabilidad 30 $\mathrm{m}^{3} / \mathrm{m}^{2} / \mathrm{h} @ 50 \mathrm{~Pa}$.

El promedio diario de concentración de MP2.5 es $6 \mu \mathrm{g} / \mathrm{m}^{3}$ más alto en la vivienda simulada con permeabilidad $10 \mathrm{~m}^{3} / \mathrm{m}^{2} / \mathrm{h} @ 50 \mathrm{~Pa}$, en comparación con aquella con permeabilidad $30 \mathrm{~m}^{3} / \mathrm{m}^{2} / \mathrm{h} @ 50 \mathrm{~Pa}$.

Si comparamos el promedio de todo el período de invierno de concentración de material particulado (ver Tabla 2), podemos ver que todas las de MP10 están por sobre $110 \mu \mathrm{g} / \mathrm{m}^{3}$, y las de MP2.5 sobre los
$58 \mu \mathrm{g} / \mathrm{m}^{3}$. Estos resultados son significativamente mayores a las Directrices de Calidad del Aire planteadas por la Organización Mundial de la Salud ${ }^{34}$, donde la primera meta establece concentraciones de $70 \mu \mathrm{g} / \mathrm{m}^{3}$ para MP10 y $35 \mu \mathrm{g} / \mathrm{m}^{3}$ para MP2.5.

La evidencia epidemiológica de la OMS establece que el incremento de $10 \mu \mathrm{g} / \mathrm{m}^{3}$ en las concentraciones diarias de MP10, puede significar un aumento de $0,5 \%$ en mortalidad. De igual manera, los estudios de Dockery y Pope ${ }^{35}$ han reportado que el incremento de $10 \mu \mathrm{g} / \mathrm{m}^{3}$ de MP10, puede aumentar en promedio un 1,4\% las muertes cardiovasculares y un 3,5\% las muertes por problemas respiratorios.

Estudios realizados en la ciudad de Temuco en relación con la calidad del aire atmosférica, se han enfocado en factores de riesgo para la salud, especialmente durante el período de invierno, cuando los niveles de concentración aumentan debido a la

34 World Health Organization, 2005.

35 Dockery y Pope, 1994. 
combustión de leña residencial ${ }^{36}$. Dichos trabajos estadísticos ${ }^{37}$ se han desarrollado en la ciudad para determinar la relación entre la polución de MP10 y sus efectos en la salud, medidos en la cantidad de muertes diarias, hospitalizaciones y visitas a salas de emergencia por enfermedades cardiovasculares, respiratorias e infecciones respiratorias agudas. Los modelos fueron desarrollados para la totalidad de la población, discriminados por edad, y analizados para los períodos de invierno y verano. Los resultados del análisis estadístico fueron reportados en Riesgo Relativo (RR) causado por el aumento diario de $100 \mu \mathrm{g} / \mathrm{m}^{3}$ de MP10. El mayor riesgo relativo para mortalidad cuando hay un incremento diario de $100 \mu \mathrm{g} / \mathrm{m}^{3}$ de MP10 en los períodos de invierno, corresponde a las enfermedades respiratorias en la población sobre 64 años de edad, con un RR de 1.175. Este mismo grupo etario arrojó el mayor RR para hospitalizaciones.

Dadas las evidencias epidemiológicas de los efectos en la salud que tiene el aumento de las concentraciones atmosféricas de MP10, se puede asumir, tal como plantea la Organización Mundial de la Salud, que un alza en las concentraciones intradomiciliarias debido a la menor permeabilidad de la vivienda, tendría el mismo efecto en la salud de la población.

36 Sanhueza et al, 2006.

37 Sanhueza et al, 2009.

\section{Conclusiones}

Los hallazgos encontrados en los modelos realizados en este estudio y en el caso hipotético presentado, ponen de manifiesto la importancia de considerar la contaminación intradomiciliaria y sus posibles efectos en la salud. El objetivo de este estudio ha sido relacionar las variables de permeabilidad de la vivienda con las concentraciones de material particulado generado por cocinas residenciales con combustión de leña. Esto, principalmente, porque implica relacionar dos políticas públicas planteadas en el plan de descontaminación atmosférico de la ciudad de Temuco.

Por un lado, el subsidio de acondicionamiento térmico está enfocado a viviendas sociales y puede reducir la permeabilidad de las viviendas y, por otro, el recambio voluntario de la cocina a leña puede tener mayores dificultades para las familias de menores recursos. Es posible que no puedan financiar el remplazo de la cocina a leña por modelos más eficientes, ya que tiene la doble función de cocinar y calefaccionar; su uso tiene arraigos culturales, y la leña es el combustible más económico y disponible en el mercado. Por lo tanto, se puede predecir que este grupo socioeconómico podría estar 
más expuesto a un aumento en la concentración de contaminantes, si se reduce la permeabilidad de la vivienda y no hay recambio de la cocina.

Los subsidios para mejorar la aislación térmica de las viviendas deben ser fuertemente promovidos, porque significan un modo adecuado para reducir la demanda energética por concepto de calefacción. Sin embargo, si seguimos los hallazgos de esta investigación, el reacondicionamiento de las viviendas debería incluir adecuadas especificaciones de ventilación natural o mecánica, a fin de reducir los altos niveles de concentración de contaminantes dentro de los hogares. La calidad del aire intradomiciliario por combustión a leña es un tema de gran importancia en Chile y requiere de más estudios e investigaciones para analizar y profundizar cómo algunas políticas públicas podrían afectar la salud de la población.

\section{Bibliografía}

AMBIENTE Consultores. Programa de inversión pública para fomentar el reacondicionamiento térmico del parque construido de viviendas. Reporte final, licitación pública 587-368-LE06. Santiago, Chile. Ministerio de Vivienda y Urbanismo. 2007(a).

AMBIENTE Consultores. Análisis técnico y económico de la aplicación de una norma de emisión para artefactos de uso residencial que combustionan con leña y otros combustibles de biomasa. Reporte final, licitación pública 1285-29-LE06. Santiago, Chile, CONAMA. 2007(b). 98 p.

CÁRDENAS, Juan Pablo. Determinación del comportamiento energitérmico de viviendas en Temuco. Concurso DIUFRO 2009. Proyecto de Investigación Científica. Temuco, Chile, Universidad de la Frontera. 2010.

CHILE Ambiente Corporación. Análisis del potencial estratégico de la leña en la matriz energética chilena. Reporte Final. Santiago, Chile, Comisión Nacional de Energía. Diciembre, 2008. 290 p.

COLLADOS, Emilio y ARMIJO, Gabriela. Impact of an energy refurbishment programme in Chile: more than energy savings. En: MUMOVIC, Dejan y SANTAMOURIS, Mat. A handbook of sustainable building design and engineering. Londres, Reino Unido, Earthscan. 2009. p 401-422. ISBN 978-1-84407-596-6.

COMISIÓN Nacional de Energía. Balance nacional de energía 2008. [En línea]. Comisión Nacional de Energía. 2008(a) [Fecha de consulta: 21 julio 2010]. Disponible en: http://www.cne.cl/ estadisticas/balances-energeticos.

COMISIÓN Nacional de Energía. Política energética: nuevos lineamientos. Transformando la crisis energética en una oportunidad. Santiago, Chile, Comisión Nacional de Energía. 2008(b).

DOCKERY, D. W y POPE, C. A. Acute respiratory effects of particulate air pollution. [En línea]. Annual Review of Public Health. 15: 107-132, mayo 1994. 
ISSN 0163-7525. Disponible en: http://dx.doi. org/10.1146/annurev.pu.15.050194.000543.

GORDON, Bruce; MACKAY, Richards y REHFUESS, Eva. Inheriting the world: the atlas of children's health and the environment. Brighton, Reino Unido, World Health Organization. 2004. 63 p. ISBN 92-4-159156-0.

INSTITUTO Nacional de Estadísticas. Censo nacional de población y vivienda 2002. [En línea]. INE. 2012. [Fecha de consulta: 26 julio 2010]. Disponible en: http://www.ine.cl/canales/chile_estadistico/censos_ poblacion_vivienda/censo_pobl_vivi.php.

MATTHEWS, Emily; PAYNE, Richard; ROHWEDER, Mark y MURRAY, Siobhan. Pilot analysis of global ecosystems: forest ecosystem. Washington, Estados Unidos, World Resources Institute. 2000. 86 p. ISBN 1-56793-459-3.

MINISTERIO de Vivienda y Urbanismo. Manual de Aplicación de Reglamentación Térmica (MART). Artículo 4.1.10 de la Ordenanza General de Urbanismo y Construcción. Santiago, Chile, Instituto de la Construcción. 2006.

MINISTERIO del Medio Ambiente. Decreto No 39, que establece una norma de emisión de material particulado para los artefactos que combustionen o puedan combustionar leña y derivados de la madera. Santiago de Chile, Ministerio del Medio Ambiente. 2012(b). 5 p.

---- Informe del estado del medio ambiente 2011. $2^{\text {a }}$ ed. Santiago de Chile, Ministerio del Medio Ambiente. 2012(a). 511 p. ISBN 978-956-7204-44-1.
MINISTERIO Secretaría General de la Presidencia. Decreto $N^{\circ} 78$, que establece un plan de descontaminación atmosférica de Temuco y Padre Las Casas. 2010. Santiago, Chile, Contraloría General de la República. 38 p.

SANHUEZA, Pedro; TORREALBA, Mónica; DÍAZ-ROBLES, Luis; SCHIAPPACASSE, Nicolas; SILVA, María y ASTETE, Teresa. Particulate air pollution and health effects for cardiovascular and respiratory causes in Temuco, Chile: a wood-smokepolluted urban area. [En línea]. Journal of Air \& Waste Management Association. 59(12): 1481-1488, diciembre 2009. ISSN 2162-2906. Disponible en: http://dx.doi.org/10.3155/1047-3289.59.12.1481.

SANHUEZA, Pedro; VARGAS, Claudio y MELLAD0, Paula. Impacto de la contaminación del aire por PM10 sobre la mortalidad diaria en Temuco. [En línea]. Revista Médica de Chile. 134(6): 754-761, junio 2006. ISSN 07176163. Disponible en: http://dx.doi.org/10.4067/ S0034-98872006000600012.

SISTEMA Nacional de Certificación de Leña. Reglamento de certificación. [En línea]. SNCL. 2012. [Fecha de consulta: 22 julio 2010]. Disponible en: http://www.lena.cl/reglamento-de-certificacion/.

UNIVERSIDAD de Chile. Diagnóstico del mercado de la leña en Chile. Santiago, Centro de Microdatos, Universidad de Chile. 2005. 185 p.

UNIVERSIDAD de la Frontera. Diseño de un programa de recambio de artefactos existentes que combustionan leña por tecnología menos contaminante, en las comunas de Temuco y Padre Las Casas. 
Reporte final, licitación pública 1285-11014CO08. Temuco, Chile, CONAMA. 2009. 244 p.

WORLD Health Organization. Addressing the links between indoor air pollution, household energy and human health. Report from Washington consultation. Geneva, Suiza, WHO Press. 2000. 56 p.
---- Air quality guidelines for particulate matter, ozone, nitrogen, dioxide and sulfur dioxide, Global update 2005, summary of risk assessment. Geneva, Suiza, WHO Press. 2005. 21 p.

---- Fuel for life: household energy and health. Geneva, Suiza, WHO Press. 2006. 42 p. ISBN 92-4-156316-8. 


\section{INFORMATIVO/NEWS BULLETIN}

